\title{
COVID-19 experience and challenges from a referral hospital in East Borneo, Indonesia: a case series
}

Kevin Aristyo, Filbert Riady Adlar, Diah Adhyaksanti

Department of Respiratory Medicine, Balikpapan General Hospital, Balikpapan, East Borneo, Borneo, Indonesia

\begin{abstract}
Coronavirus disease 2019 (COVID-19) is a multifaceted disease and pandemic control requires a collaborative approach between various stakeholders from frontline doctors to governing bodies. Here we report the challenges faced in early COVID-19 cases that were admitted to Balikpapan General Hospital, East Borneo, Indonesia. We highlighted the insufficiencies in implementing the regulations endorsed by Indonesian Government in low resource settings.
\end{abstract}

Keywords: COVID-19; resource limited; subclinical; length of stay; Indonesia

\section{Introduction}

Coronavirus disease 2019 (COVID-19) pandemic has reached Indonesia at the beginning of March 2020 and confirmed cases have been reported throughout all provinces. ${ }^{1}$ Several hospitals have been designated as the referral centers for COVID-19 albeit not equipped with essential diagnostic modalities such as computed tomography (CT) scan and/or reversetranscriptase polymerase chain reaction (RT-PCR). ${ }^{1}$ Furthermore, delay in diagnosis and misdiagnosis might be attributed to complex nature of the disease and its interaction with the host immune response. ${ }^{2}$ The Severe Acute Respiratory Syndrome Coronavirus 2 (SARS-CoV2) is capable of infecting multiple organs of the body through binding with the angiotensinconverting enzyme 2 (ACE2) receptor. ${ }^{2}$ This contributed to the diverse reported clinical 
manifestations of COVID-19, ranging from asymptomatic to subclinical involvement of various body systems to acute respiratory distress syndrome (ARDS). ${ }^{3}$ Thus diagnosing COVID-19 in a resource-limited setting is challenging. This case series highlights our experience with COVID-19 from a first level referral hospital in East Borneo, Indonesia.

\section{Case Report:}

From March to June 2020, 5 patients diagnosed with COVID-19 were admitted to Balikpapan General Hospital, East Borneo, Indonesia. According to WHO's case definition, cases 1 and 2 were imported cases while the remaining 3 were locally transmitted. There were 3 males and 2 females included in this report. The main clinical characteristics and presenting symptoms of these cases can be seen in tables 1 and 2 respectively. Pulmonary physical examinations (PE) of all patients were unremarkable. Initial investigations included a complete blood count, SARS-CoV-2 antibody rapid test and chest X-ray (figure 2). SARS-CoV-2 antibody (IgM) rapid test was reactive in all cases. However, erythrocyte sedimentation rate (ESR) and Creactive protein (CRP) examinations were limited to some cases. All cases had fully recovered and were discharged.

Schematic presentation of important events for each case is shown in figure 1. Case 1 was a 57-year-old man diagnosed with COVID-19 on Mar 22, 2020 (illness day 5), 10 days after attending a religious event at Bogor, East Java with his 53-year-old wife (case 2). Both cases exhibited influenza-like symptoms and no abnormalities on chest x-ray. Initial routine blood examination performed on case 1 was normal while case 2 had mild leukopenia, differential count indicating shift to the right, and elevated ESR. Both cases experienced no clinical deterioration and recovered fully. 
Table 1. Main characteristics of patients at hospital admission

\begin{tabular}{|c|c|c|c|c|c|}
\hline & Case 1 & Case 2 & Case 3 & Case 4 & Case 5 \\
\hline Age, years & 57 & 53 & 45 & 61 & 20 \\
\hline Sex & Male & Female & Male & Female & Male \\
\hline Past medical history & None & None & None & Asthma & None \\
\hline Length of stay, days & 33 & 33 & 23 & 34 & 13 \\
\hline Diagnosis date & Mar 28, 2020 & Mar 28, 2020 & Apr 10, 2020 & May 5, 2020 & June 5,2020 \\
\hline $\begin{array}{l}\text { Turnaround time for } \\
\text { PCR, days }\end{array}$ & 6 & 6 & 8 & ND & ND \\
\hline \multicolumn{6}{|l|}{ Laboratory parameters } \\
\hline Hemoglobin, g/dL & 14.7 & 13.1 & 15.7 & 11.7 & 15.6 \\
\hline Hematocrit, $\%$ & 43.1 & 40.6 & 46 & 33.3 & 45.5 \\
\hline Leukocyte, $10^{3} / \mathrm{uL}$ & 8.9 & 3.3 & 6.0 & 12.3 & 6.2 \\
\hline Neutrophil, \% & 54 & 37.7 & 60.8 & 66 & 83.5 \\
\hline Lymphocyte, \% & 36 & 52 & 29.7 & 25 & 5.18 \\
\hline $\begin{array}{l}\text { Absolute Lymphocyte } \\
\text { cells/ uL }\end{array}$ & 3204 & 1716 & 1782 & 3075 & 321.2 \\
\hline NLR & 1.5 & 0.7 & 2.1 & 2.64 & 16.1 \\
\hline Platelet count, $10^{3} / \mathrm{uL}$ & 369 & 186 & 235 & 292 & 234 \\
\hline PLR & 115.16 & 108.39 & 131.87 & 94.95 & 728.60 \\
\hline ESR, mm/hour & 11 & 47 & ND & ND & ND \\
\hline $\mathrm{CRP}, \mathrm{mg} / \mathrm{L}$ & ND & ND & 65 & 47 & ND \\
\hline
\end{tabular}

$\mathrm{ND}=$ not determined, NLR = Neutrophil-lymphocyte ratio, PLR = Platelet-lymphocyte ratio, ESR = Erythrocyte sedimentation rate, $\mathrm{CRP}=\mathrm{C}$-Reactive Protein $(\mathrm{CRP})$

Table 2. Presenting clinical features of patients

\begin{tabular}{|c|c|c|c|c|c|c|c|c|c|c|}
\hline Case & Fever & $\begin{array}{l}\text { Sore } \\
\text { Throat }\end{array}$ & $\begin{array}{l}\text { Dry } \\
\text { Cough }\end{array}$ & $\begin{array}{l}\text { Productive } \\
\text { Cough }\end{array}$ & Headache & Nausea & Diarrhea & Fatigue & Anosmia & Ageusia \\
\hline 1 & + & & + & & + & + & & & + & + \\
\hline 2 & + & + & + & & + & + & & + & + & + \\
\hline 3 & + & & & + & + & + & + & + & + & + \\
\hline 4 & & + & + & & & & & & + & + \\
\hline 5 & & & & & & & & & & \\
\hline
\end{tabular}

Case 3 was a 45-year-old man presented with chief complaint of diarrhea and prior history of fever and cough. He attended a local wedding reception 14 days before symptoms appeared and was diagnosed with COVID-19 on Apr 1, 2020 (illness day 8). On admission, vital signs were stable and bowel movements were markedly increased otherwise PE was unremarkable. CRP was elevated and signs of bronchopneumonia \& cardiomegaly were seen in chest X-ray. He became asymptomatic from the sixth day following admission until discharged. 


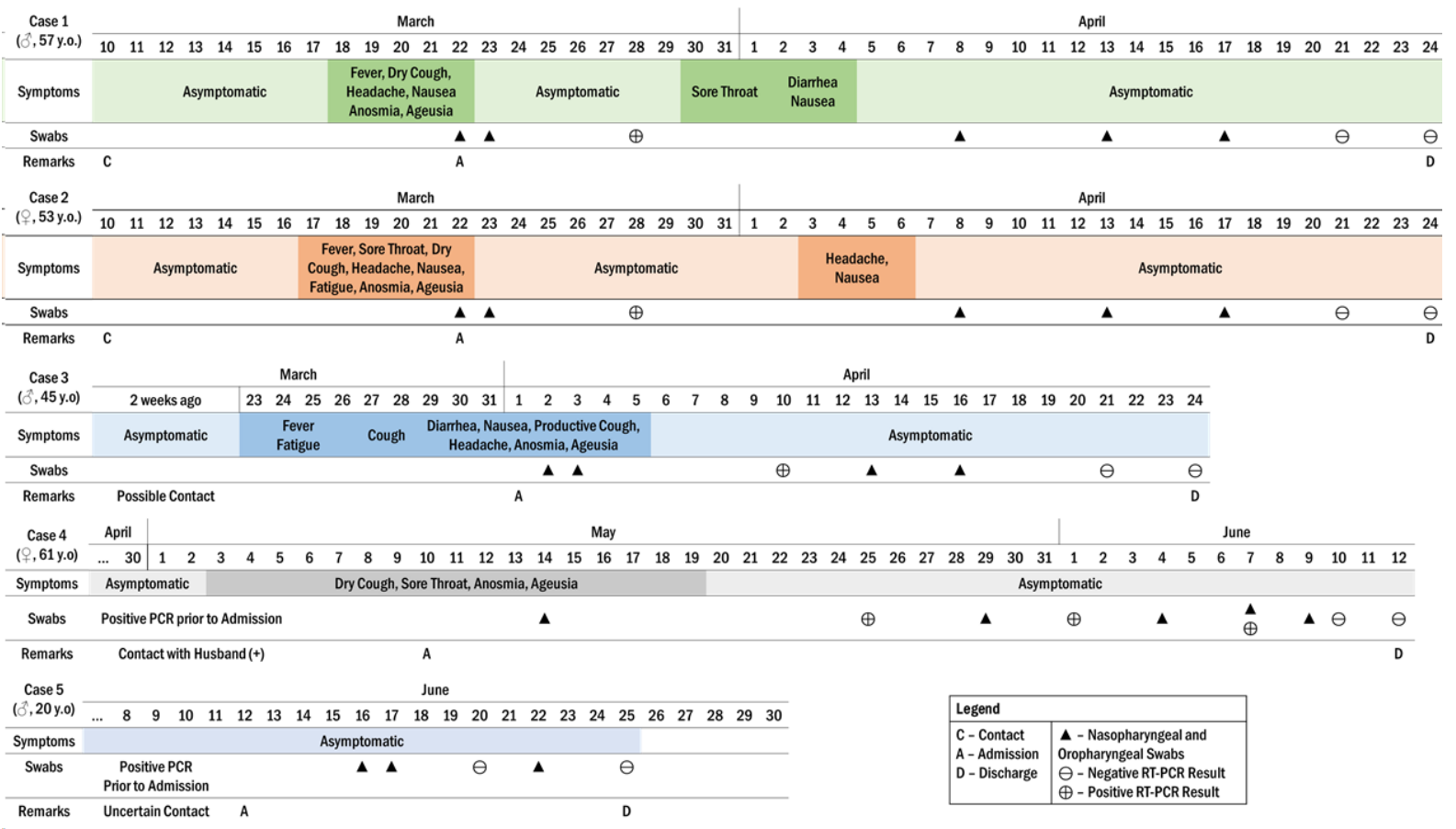

Figure 1. Schematic diagram of six cases of COVID-19 in Balikpapan, East Borneo,

Indonesia.

Cases 4 and 5 were transported to our hospital with positive nasopharyngeal PCR swab prior to admission. Thus, the turnaround time for PCR results for these two was not determined. Case 4, 61-year-old woman, had close contact with a confirmed COVID-19 patient; her husband who was hospitalized at a different hospital. She had symptoms of cough and sore throat, and history of asthma. Initial laboratory results showed mild leukocytosis and elevated CRP. Chest X-ray was within normal limits. After 9 days following admission, she became asymptomatic until discharged. Case 5, 20-year-old male, worked as a cashier at a local store and recalled no specific contact with confirmed COVID-19 cases. He was asymptomatic throughout the hospital stay and was discharged after 13 days of hospitalization. Laboratory results showed differential count shifting to the left with high neutrophil-lymphocyte ratio (NLR) of 16.1. Initial chest X-ray showed aspects of bronchitis. 


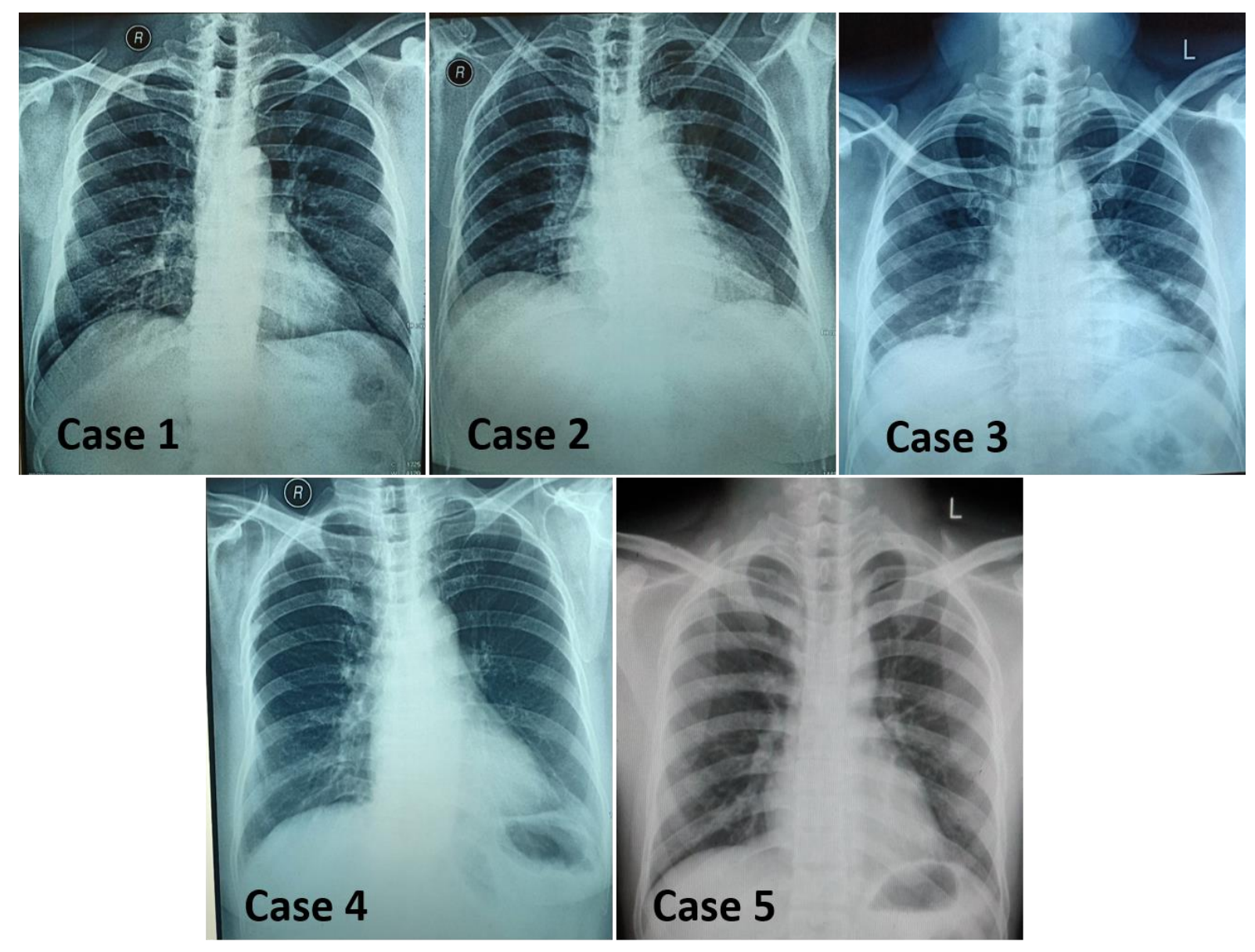

Figure 2. X-ray findings and interpretations of the 5 cases. Case 1: X-ray within normal limits. Case 2: Cardiomegaly with aortic dilation and elongation. Lungs within normal limits. Case 3: Bronchopneumonia. Cardiomegaly with aortic dilation and elongation. Case 4: X-ray within normal limits. Case 5: Aspects of bronchitis.

\section{Discussion}

In this case series, we illustrated the subclinical nature of COVID-19 in patients admitted to our hospital; four paucisymptomatic cases and one asymptomatic case.

Subjective anosmia and ageusia were present in four out of five cases. Many studies have reported both symptoms as the newly emerging presenting clinical features of COVID-19 as it has been hypothesized that the virus could cause olfactory nerve neuropathy. ${ }^{4}$ Few case reports have shown that patients with positive COVID-19 with anosmia and/or ageusia as the sole 
symptoms. ${ }^{5,6}$ Data of COVID-19 cases from a residential treatment center in the Republic of Korea showed anosmia was present in $26.1 \%$ of cases while $22.6 \%$ had ageusia and $19.1 \%$ had both. ${ }^{6}$ Objective assessment of sensory dysfunction in COVID-19 by Varia et al showed $61.1 \%$ had evident decline in olfactory or gustatory function compared to $73.6 \%$ from subjective complaints. ${ }^{4}$ Therefore, physicians should be aware of the decline in chemosensory function as possible symptoms of COVID-19. In practice this suggests that subjective evaluation can be a useful screening tool for COVID-19. The early screening tool published by Indonesia Ministry of Health did not include anosmia and ageusia as possible criteria. ${ }^{7}$ This might lead to delay in diagnosis or misdiagnosis.

Diarrhea was the presenting problem in case 3 and was initially not suspected of COVID-19 if not for the prior history of possible contact. The SARS-CoV-2 rapid test was reactive on admission and afterwards assigned in the isolation ward. Gastrointestinal symptoms are also an often mislooked complaint of COVID-19 infection. ${ }^{8}$ In the study by Han et al, digestive symptoms were present in $56.7 \%$ in COVID-19 cases and of which $19.4 \%$ had diarrhea as its first symptom. ${ }^{8}$ Delayed diagnosis of COVID-19 can potentially be detrimental to other patients and healthcare providers. Unsuspecting physicians might put COVID-19 patients with mild or atypical symptoms in the general ward which can increase the risk of nosocomial COVID-19 infection. ${ }^{9}$

The mean interval $( \pm \mathrm{SD})$ length of stay $(\mathrm{LoS})$ was $27 \pm 8.2$ days and median LoS was 33 days (IQR:18-33.5). A systematic review of 52 studies calculated the median hospital LoS due to COVID-19 was 14 days (IQR:14-19) in China and 7 (IQR:3-9) outside of China. ${ }^{10}$ The increased LoS of COVID-19 patients in our hospital was due to the lack of RT-PCR facility in Borneo. The nasopharyngeal swab samples had to be transported to Surabaya on the Island of Java which was not only time consuming, but also might have compromised the sample's viability. The mean $( \pm \mathrm{SD}) \mathrm{RT}-\mathrm{PCR}$ turnaround time in our first 3 cases was $6.7 \pm 1.2$ days. 
Previous WHO recommendation of two negative RT-PCR tests as the discharge criteria has been difficult in Indonesia where the testing capacity was overburdened during the initial stage of the Pandemic. Prolonged LoS decreased bed availability for other potential COVID-19 patients in need.

Current scoring used such as the COVID-19 early warning score (COVID-19 EWS) relied on the result of CT scan. ${ }^{11}$ Computed Tomography (CT) scan was able to detect lung abnormalities even in asymptomatic patients which will facilitate the early identification of the diseases however CT scan was not available in our hospital. Thus, a robust and accurate scoring, validated with Indonesian population, relying on basic parameters readily available was desperately needed to assist physicians in this kind of setting.

To our knowledge, this is the first COVID-19 case series reporting early cases from Borneo Island, Indonesia. We illustrated the diagnostic value of ageusia, anosmia, and diarrhea in COVID-19. Our study showed a notably longer LoS of COVID-19 patients compared to other countries due to lack of diagnostic capabilities in particular centers and the initial WHO criteria for discharge. There is a knowledge gap for a new COVID-19 scoring system without utilization of CT scan in low resource settings. Here we presented subclinical patients might exhibit non-typical NLR and PLR values which are not correlated to clinical severity. We highlighted that by improving the COVID-19 screening tools to accommodate diverse subclinical COVID-19 manifestations can optimize early detection of COVID-19 in resource limited settings such as Indonesia.

\section{Disclosure Statement}

The authors declare that they have no competing interests. 


\section{References}

1. Djalante, R. et al. Review and analysis of current responses to COVID-19 in Indonesia: Period of January to March 2020. Prog. Disaster Sci. 6, 100091 (2020).

2. Zhai, P. et al. The epidemiology, diagnosis and treatment of COVID-19. Int. J. Antimicrob. Agents 55, 105955 (2020).

3. Singhal, T. A Review of Coronavirus Disease-2019 (COVID-19). Indian J. Pediatr. 87, $281-286(2020)$.

4. Vaira, L. A. et al. Objective evaluation of anosmia and ageusia in COVID -19 patients: Single-center experience on 72 cases. Head Neck 42, 1252-1258 (2020).

5. Zhang, Q., Shan, K. S., Abdollahi, S. \& Nace, T. Anosmia and Ageusia as the Only Indicators of Coronavirus Disease 2019 (COVID-19). Cureus 12,.

6. Noh, J. Y. et al. Asymptomatic infection and atypical manifestations of COVID-19: Comparison of viral shedding duration. J. Infect. S0163445320303108 (2020) doi:10.1016/j.jinf.2020.05.035.

7. Ministry of Health, I. Guideline for prevention and management of coronavirus disease19. (2020).

8. Han, C. et al. Digestive Symptoms in COVID-19 Patients With Mild Disease Severity: Clinical Presentation, Stool Viral RNA Testing, and Outcomes. Am. J. Gastroenterol. 115, 916-923 (2020).

9. Risk of nosocomial transmission of coronavirus disease 2019: an experience in a general ward setting in Hong Kong. https://www.ncbi.nlm.nih.gov/pmc/articles/PMC7128692/.

10. Rees, E. M. et al. COVID-19 length of hospital stay: a systematic review and data synthesis. medRxiv 2020.04.30.20084780 (2020) doi:10.1101/2020.04.30.20084780.

11. Song, C.-Y., Xu, J., He, J.-Q. \& Lu, Y.-Q. COVID-19 early warning score: a multiparameter screening tool to identify highly suspected patients. 
http://medrxiv.org/lookup/doi/10.1101/2020.03.05.20031906 (2020) doi:10.1101/2020.03.05.20031906. 